\title{
Diabetes Perturbs Bone Microarchitecture and Bone Strength through Regulation of Sema3A/IGF-1/ $\beta$-Catenin in Rats
}

\author{
Rufeng Ma Lili Wang $^{\mathrm{a}}$ Baosheng Zhao ${ }^{\mathrm{b}}$ Chenyue Liu ${ }^{\mathrm{c}}$ Haixia Liu ${ }^{\mathrm{a}}$ \\ Ruyuan Zhu ${ }^{a}$ Beibei Chen ${ }^{a}$ Lin Lia Dandan Zhao ${ }^{d}$ Fangfang Mo ${ }^{d}$ Yu Lia \\ Jianzhao Niu ${ }^{a}$ Guangjian Jiang ${ }^{d}$ Min Fu ${ }^{e}$ Dieter Bromme ${ }^{f}$ Dongwei Zhang ${ }^{d}$ \\ Sihua Gao ${ }^{d}$

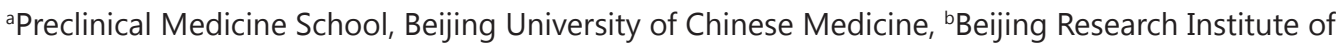 \\ Traditional Chinese medicine, Beijing University of Chinese Medicine, 'Chinese Material Medica School, \\ Beijing University of Chinese Medicine, dDiabetes Research Center, Beijing University of Chinese

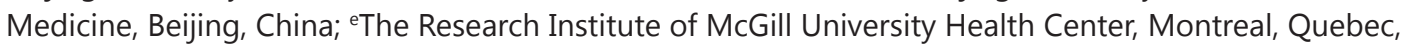 \\ fOral Biological Medicinal Science, University of British Columbia, Vancouver, Canada
}

\section{Key Words}

Diabetic rats $•$ Sema3A $~ \beta$-catenin - Insulin-like growth factor-1 (IGF) $\bullet$ Peroxisome proliferatoractivated receptor $\gamma($ PPAR $\gamma) \cdot$ Cathepsin $\mathrm{K}$

\begin{abstract}
Purpose: Increasing evidence supported that semaphorin 3A (Sema3A), insulin-like growth factor (IGF)-1 and $\beta$-catenin were involved in the development of osteoporosis and diabetes. This study is aimed to evaluate whether Sema3A/IGF-1/ $\beta$-catenin is directly involved in the alterations of bone microarchitecture and bone strength of diabetic rats. Methods: Diabetic rats were induced by streptozotocin and high fat diet exposure. Bone microarchitecture and strength in the femurs were evaluated by micro-CT scanning, three-point bending examination and the stainings of $\mathrm{HE}$, alizarin red $\mathrm{S}$ and safranin $\mathrm{O} /$ fast green, respectively. The alterations of lumbar spines microarchitecture were also determined by micro-CT scanning. Western blot and immunohistochemical analyses were used to examine the expression of Sema3A, $\beta$-catenin, IGF-1, peroxisome proliferator-activated receptor $\gamma$ (PPAR $\gamma$ ) and cathepsin $\mathrm{K}$ in rat tibias. Results: Diabetic rats exhibited decreased trabecular numbers and bone formation, but an increased trabecular separation in the femurs and lumbar spines. Moreover, the increased bone fragility and decreased bone stiffness were evident in the femurs of diabetic rats. Diabetic rats also exhibited a pronounced bone phenotype which manifested by decreased expression of Sema3A, IGF- 1 and $\beta$-catenin, as well as increased expression of cathepsin $\mathrm{K}$ and PPARY. Conclusions: This study suggests that diabetes could perturb bone loss through the Sema3A/IGF-1/ $\beta$-catenin pathway. Sema3A deficiency in bone may contribute to upregulation of PPARY and cathepsin K expression, which further disrupts bone remodeling in diabetic rats.

R. Ma, L. Wang and B. Zhao contributed equally.

Dongwei Zhang, MD \& PhD and Sihua Gao, MD

Associate Professor of Diabetes Research Center, Beijing University of Chinese Medicine Beijing 100029 (PR China), Diabetes Research Center, Beijing University of Chinese Medicine Beijing 100029 (PR China)

E-Mail dongwei1006@gmail.com / dongwei1006@gmail.com / gaosihua1216@163.com
\end{abstract}

KARGER 


\section{Cellular Physiology Cell Physiol Biochem 2017;41:55-66 \begin{tabular}{l|l|l} 
and Biochemistry.1159/000455936 & $\begin{array}{l}\text { ( ) 2017 The Author(s). Published by S. Karger AG, Basel } \\
\text { www.karger.com/cpb }\end{array}$
\end{tabular} \\ Ma et al.: Sema3A and Diabetic Osteoporosis Rats}

\section{Introduction}

Diabetic osteoporosis is a chronic bone metabolic disease induced by diabetes mellitus (DM), and characterized by increased risk of osteoporotic fracture and deterioration of bone microarchitecture $[1,2]$. In the past decades, the incidence of diabetic osteoporosis is increasing with the epidemic spread of diabetes worldwide [2]. Hyperglycemia may negatively affect bone metabolism through disrupting the delicate balance between osteoblastogenesis and osteoclastogenesis [3]. In addition, DM also increases adipogenesis in the bone marrow, and further expands the marrow cavity and narrows the cortical envelope [3,4]. Moreover, in postmenopausal women with type 2 DM, decreased serum level of IGF-1 are positively associated with vertebral fractures [5]. Meanwhile, the activity of cathepsin K, a marker of bone resorption, is increased in diabetic rats [6] as well as in diabetic postmenopausal woman [7]. Furthermore, the relative abundance of PPAR $\gamma$ expression in metabolic disorders promotes mesenchymal stem cells differentiation into adipocytes at the cost of osteoblasts [8-10].

Semaphorin 3A (Sema3A), originally identified as an axonal guidance chemorepellent with an essential role in neuronal development and regeneration following injury [11], has been reported to act as a local regulator of bone remodeling through promoting bone formation by inducing osteoblast differentiation via the stimulation of the canonical Wnt/ $\beta$-catenin signaling pathway, and inhibiting bone resorption via suppressing RANKLinduced osteoclast differentiation through the inhibition of immunoreceptor tyrosine-based activation motif signals [12-15]. Thus, increasing the expression of Sema3A contributes to the regeneration of bone defects and titanium implant fixations in ovariectomized (OVX) animals $[16,17]$ as well as the improvement of osseointegeration in type 2 DM rats [18]. However, whether Sema3A and the associated proteins of $\beta$-catenin and IGF- 1 are involved in alterations of bone microstructure and strength of diabetic rats remains unclear.

Therefore, in the current study we attempted to investigate the expression of Sema3A, $\beta$-catenin and IGF-1 in the bones of streptozotocin (STZ) and high fat diet (HFD) induced diabetic rats, and as well as its related alterations in bone microarchitecture and biomechanical strength. The results demonstrated that Sema3A expression was significantly decreased which may be associated with irregular bone microarchitecture and reduced bone quality. Meanwhile, the expression of $\beta$-catenin and IGF- 1 was decreased, and the expression of cathepsin $\mathrm{K}$ and PPAR $\gamma$ was increased in the bones of diabetic rats. The results suggest that diabetes may disturb the bone metabolism through regulation of the Sema3A/IGF-1/ $\beta$ catenin signaling pathway.

\section{Materials and Methods}

\section{Chemicals and antibodies}

Alizarin red S, Safranin 0 and fast green were purchased from Sigma-Aldrich (St. Louis, MO, USA). Antibodies against IGF-1 (sc-9013) and Sema3A (sc-28867) were from Santa Cruz Biotechnology (Dallas, TX, USA). Antibodies against $\beta$-catenin (9562S) and phosphor- $\beta$-catenin (9561) were purchased from Cell Signaling Technology (Danvers, MA, USA). Antibodies against cathepsin K antibody (ab19027) and PPAR $\gamma$ (ab191407) were purchased from Abcam (Cambridge, UK). All the chemicals, except specially identified, were obtained from Sino pharm Beijing Chemical Reagents Co. Ltd (Beijing, China).

\section{Animal models establishment}

6 to 8 weeks old female SD rats (220 \pm 10 g) were purchased from China Huafukang Animal Technology Co. Ltd. (License No.: SCXK (Beijing) 2011-0004, Beijing, China). The animals were housed at clean level conditions (certification number SCXK (Jing) 2011-0024) at Beijing University of Chinese Medicine (BUCM) with the temperature of $22 \pm 1^{\circ} \mathrm{C}$, humidity of $55 \pm 5 \%$, and a $12 \mathrm{~h}$ light/dark cycle. All rats were allowed free access to tap water and food. The study protocol was approved by the animal care committee of BUCM, China. 


\section{Cellular Physiology Cell Physiol Biochem 2017;41:55-66 \begin{tabular}{l|l|l|}
\hline DOI: 10.1159/000455936 & $\begin{array}{l}\text { C) } 2017 \text { The Author(s). Published by S. Karger AG, Basel } \\
\text { www.karger.com/cpb }\end{array}$
\end{tabular} \\ Ma et al.: Sema3A and Diabetic Osteoporosis Rats}

After one week of acclimation, the rats were divided into two groups, normal control group and diabetic group. The rats in the diabetic group were fed with HFD (20\% sucrose, $2.5 \%$ cholesterol, $10 \%$ lard, $1 \%$ sodium cholic acid, and $66.5 \%$ (w/w) standard chow) for one month. Then STZ (30 mg/kg) were intraperitoneally injected into the rats for once. Three days after injection, 10 of the SD rats, whose blood glucose values reached $11.1 \mathrm{mM}$ or higher, were selected as the diabetic model group and followed by HFD feeding for additional two months. The same numbers of rats in the control group were fed with the normal diet. The rats in both groups had free access to the tap water and food. After three months of administration, all rats were anesthetized with pentobarbital sodium ( $1 \%$ sodium pentobarbital, $0.4 \mathrm{ml} / 100 \mathrm{~g}$, i.p.). The bilateral femurs and tibias were harvested and stored at $-80^{\circ} \mathrm{C}$ for further analyses.

The stainings of hematoxylin/eosin (HE), alizarin red $S$ and safranin $0 /$ fast green

The right femurs of rats were first immersed in 10\% neutral formalin for $72 \mathrm{~h}$ and then decalcified in 15\% neutral EDTA buffer for three months. The decalcified femurs were defatted and dehydrated with xylene and graded ethanol (50\%-100\%), and embedded in paraffin. Sections of approximately 4 $\mu \mathrm{m}$ thickness were used for the stainings of HE, alizarin red S and safranin $0 /$ fast green. HE staining was conducted according to routine protocol [19].

Alizarin red S staining was performed according to the following procedure. Briefly, the dehydrated slides were stained with alizarin red S solution for $2 \mathrm{~min}$. Then the slides were quickly dipped into acetone and acetone xylene (50/50), each for 20 s, respectively.

Safranin 0/fast green staining was performed as follows. Briefly, the dehydrated slides were stained with hematoxylin solution for $5 \mathrm{~min}$ and differentiated with $1 \% \mathrm{HCl}$-EtOH for 2 dips, then sequentially stained with $0.05 \%$ fast green for 5 min and $0.1 \%$ safrain 0 for 5 min.

After staining, the mounted slides were observed and photographed using an Olympus BX53 fluorescence microscope (Tokyo, Japan). The tissue sections were evaluated for microarchitectural changes, including the structure and morphology of trabecular bone, lipid droplets, and the thickness of the epiphyseal plate. The relative interest of density (IOD) values of alizarin red S staining and safranin $0 /$ fast green staining were quantified using the Image Pro Plus 6.0 software.

\section{Micro-computed tomography (micro-CT) scanning}

Cone-beam x-ray micro-CT ( $\mu$ CT; Viva CT40, Scanco medical AG, Switzerland) was used to take microCT images of left femurs ( $0.5 \mathrm{~cm}$ below the femoral head) and second lumbar vertebrae. Parameters for bone content, bone microstructure, and structure mode were measured sequentially. Three-dimensional images were constructed and analyzed based on the micro-CT images using a GE Healthcare MicroView software application. The measured parameters included trabecular number (Tb.N), trabecular thickness (Tb.Th), structure model index (SMI), connectivity density (Conn.D), trabecular separation (Tb.Sp), and bone volume per trabecular bone volume (BV/TV).

Bone biomechanic strength assay

After scanning, the left femur was taken for three point bending examination by electronic universal testing machine (Shenzhen Reger Instrument Co. Ltd., Model RGWF4005, China). The shaft of the femur was fixed between two supporting points, with a distance of $20 \mathrm{~mm}$. A load was vertically applied to the femoral midshaft, with a displacement speed of $0.01 \mathrm{~mm} / \mathrm{s}$ at $100 \mathrm{~Hz}$ until the femoral shaft is fractured. The ultimate load, bending strength, and elastic modulus of the femurs were recorded.

Immunohistochemical (IHC) staining

$5 \mu \mathrm{m}$ longitudinal sections of the paraffin embedded femurs were kept at $60{ }^{\circ} \mathrm{C}$ for $24 \mathrm{~h}$ in the oven and then followed by deparaffinzing with xylene and hydrating with an ethanol gradient (100\%-70\%). After successively incubating with antigen retrieval solution (Shanghai ShunBai Biotechnology Company; Shanghai, China) and $3 \% \mathrm{H}_{2} \mathrm{O}_{2}$ for $30 \mathrm{~min}$, the slides were rinsed and incubated with the primary antibody [Sema3A (1:200), IGF-1 (1:50), $\beta$-catenin (1:200), phosphor $\beta$-catenin (1:200), PPAR $\gamma(1: 200)$ and cathepsin $\mathrm{K}(1: 200)]$ overnight at $4{ }^{\circ} \mathrm{C}$. For negative controls, the primary antibodies were replaced by non-immunized serum. The next day, the slides were rinsed and incubated with the corresponding secondary antibody (Beijing Biosynthesis Biotechnology Co. Ltd.; Beijing, China) for 30 min followed by DAB and hematoxylin staining, respectively. The slides were then examined and photographed using an Olympus BX53 fluorescence microscope. The DAB staining was analyzed by Image Pro Plus 6.0 software and expressed as IOD value. 


\section{Cellular Physiology Cell Physiol Biochem 2017;41:55-66

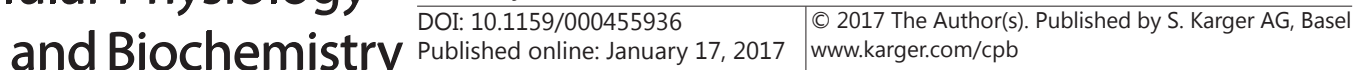 \\ Ma et al.: Sema3A and Diabetic Osteoporosis Rats}

Western blot

After the tibias and femurs were crushed in liquid nitrogen, the protein was extracted using the bone tissue protein extraction kit (Shanghai BestBio Science Biotechnology; Shanghai, China). $80 \mu \mathrm{g}$ of the protein sample was loaded into $10 \%$ or $15 \%$ of SDS-PAGE gel and transferred onto nitrocellulose blotting membrane. Afterwards, the nitrocellulose membrane was blocked with 5\% non-fat dry milk and incubated with the appropriate primary antibody [SEMA3A (1:500), IGF-1 (1:1000), $\beta$-catenin (1:1000), phosphor$\beta$-catenin (1:1000), PPAR $\gamma$ (1:1000) and cathepsin K (1:1000)], followed by the corresponding secondary antibody. Then, the signal was visualized with high-sensitivity ECL luminous liquid and the images were captured using the Azure Bioimaging system (Azure C500; California, USA). The gray values of the blots were quantified using the Image Pro Plus 6.0 software, and normalized with the blots of corresponding $\beta$-actin (1:5000) as the internal control.

\section{Statistical analysis}

All results were expressed as mean \pm standard deviation (SD). One way ANOVA was performed between groups using Prism 6.0 (GraphPad, La Jolla, USA) when homogeneity of variance and normality were met. Otherwise, Dunnett's T3 and nonparametric tests were conducted, respectively. A p-value of less than 0.05 was set as statistical significance.

\section{Results}

The alterations of fasting blood glucose levels in diabetic rats

After 3 months of treatment, the fasting blood glucose levels were measured by a glucometer. The glycaemia in the diabetic group rats $(20.21 \pm 0.92 \mathrm{mmol} / \mathrm{L})$ was significantly higher than that in the normal control group $(4.558 \pm 0.19 \mathrm{mmol} / \mathrm{L})(\mathrm{p}<0.05)$, suggesting that the rats exposed to STZ and HFD had a diabetic phenotype.

\section{Pathological bone morphology alterations in diabetic rats}

As shown in Fig. 1A, HE staining revealed a dense and regular meshwork of trabecular bone in the femurs of control rats. In contrast, the trabecular bone in the femurs of diabetic rats became thinner and irregular, and displayed a loss of regular mesh structure. Furthermore, the lipid droplets were much more evident in the proximal femurs in diabetic rats than in control rats.

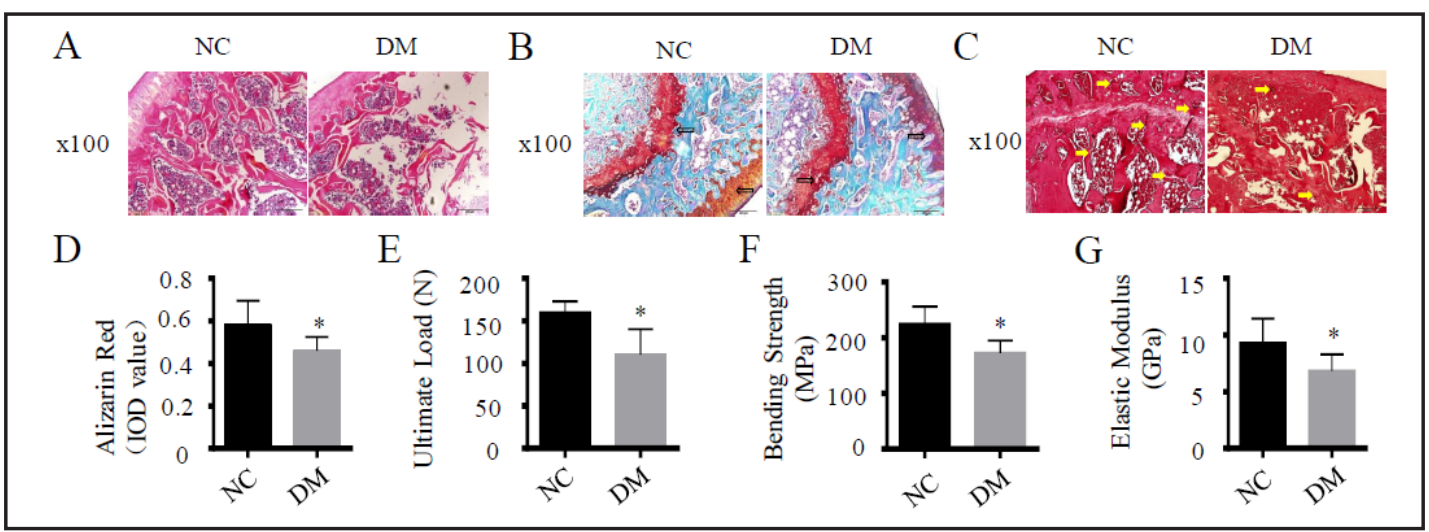

Fig. 1. Diabetes impeded bone formation and strength in rat femurs. (A) HE staining showed that the trabecular bone became thinner and loss of regular mesh structure in diabetic rats. (B) Safranin 0/fast green staining showed that the thickness of glycosaminoglycans (GAGs, red color) was decreased in the bones of diabetic rats. Alizarin red S staining (C) and its analyses (D) showed that the distribution and area of calcified nodules (yellow arrow) were significantly decreased in the femurs of diabetic rats. Three-point bending examination results of the ultimate load (E), bending strength (F), and elastic modulus (G) showed that bone fragility was increased, and bone stiffness was decreased in the femurs of diabetic rats. Data are presented as mean \pm SD. ${ }^{*} \mathrm{p}<0.05$ compared with NC. NC denotes the control rats, DM denotes diabetic rats, N=9. 
Fig. 2. Diabetes perturbed bone microarchitecture 0.5 $\mathrm{cm}$ below the femoral head) in rat femurs. Representative two-dimensional microCT images (A) of femurs and the quantification data (BG) including trabecular separation (Tb.Sp), structure model index (SMI), bone volume /tissue volume (BV/ TV), connection density (Conn.D), trabecular thick-

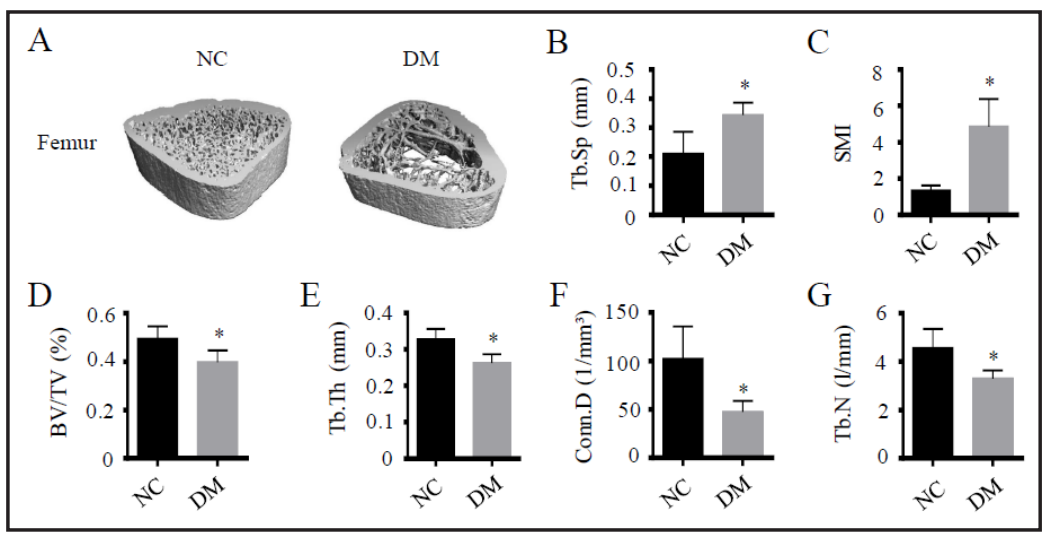
ness (Tb.Th) and trabecular number (Tb.N). Data are presented as mean \pm SD. ${ }^{*} \mathrm{p}<0.05$ compared with NC. $\mathrm{NC}$ denotes the control rats, $\mathrm{DM}$ denotes diabetic rats, $\mathrm{N}=6$.

Fig. 3. Diabetes perturbed bone microarchitecture in rat lumbar vertebraes. Representative two-dimensional micro-CT images (A) and the quantification data (B-G) including trabecular separation (Tb.Sp), structure model index (SMI), bone volume /tissue volume (BV/TV), trabecular thickness (Tb.Th), connecti-
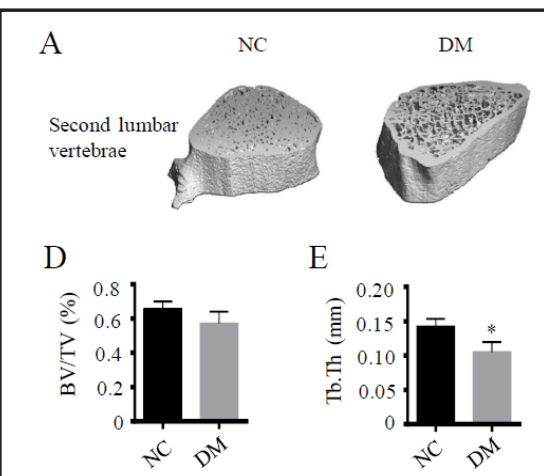

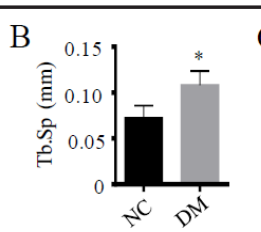

$\mathrm{E}$

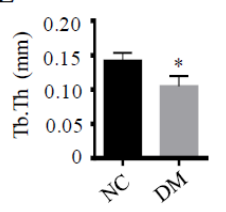

F

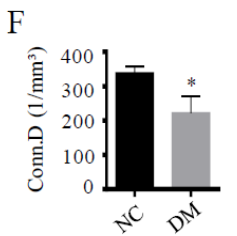

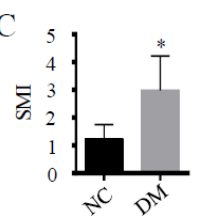

G

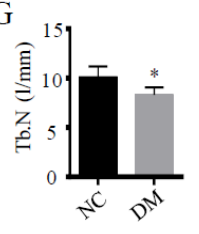

on density (Conn.D) and trabecular number (Tb.N). Data are presented as mean \pm SD. ${ }^{*} \mathrm{p}<0.05$ compared with control. ${ }^{*} \mathrm{p}<0.05$ compared with NC. NC denotes the control rats, DM denotes diabetic rats, $\mathrm{N}=6$.

Glycosaminoglycans (GAGs) are naturally-occurring carbohydrates found in cartilage which bind to hydroxyapatite and contribute to osteoblast differentiation [20-22]. To further observe the pathological changes, the stainings of safranin $0 /$ fast green and alizarin red $S$ were used to examine the epiphyseal structure in femoral heads and osteoblastic differentiation. As shown in Fig. 1B, the red color represents the staining of GAGs by safranin 0 . The thickness of cartilage adjacent to the growth plate was reduced in the femoral heads of diabetic rats when compared to that of the control group, showing that diabetes may impede osteoblast differentiation and bone formation.

During osteoblast differentiation, calcium ions are precipitated as calcium salts, which react with alizarin red S and produce deep red "calcified nodules" in the bone. The area of calcified nodules reflects the activation of osteoblasts and bone formation rate [23]. As shown in Figs. 1C and D, the distribution and extent of calcified nodules were even in the femurs of control rats. In diabetic rats, the area of calcified nodules (IOD value) was significantly decreased and calcified nodules became scantly distributed $(\mathrm{p}<0.05)$. These results indicated that bone mineralization was reduced in diabetic rats.

The alterations of bone biomechanical properties in diabetic rats

As shown in Figs. $1 \mathrm{E}$ to $1 \mathrm{G}$, three-point bending examination demonstrated that the ultimate load, bending strength and elastic modulus were significantly reduced in the femurs of diabetic rats when compared with that of control rats $(\mathrm{p}<0.05)$. The results indicated that bone fragility was markedly increased, and bone stiffness was decreased when diabetes was induced in rats. 
Fig. 4. Diabetes diminished Sema3A expression in the bones of diabetic rats. Immunohistochemical staining (A) and western blot images (C) and the analyses (B \& D) of Sema3A level in the tibias of control (NC) and diabetic (DM) rats. Data are presented as mean \pm SD. ${ }^{*} \mathrm{p}<$ 0.05 compared with $\mathrm{NC}$, $\mathrm{N}=6$.
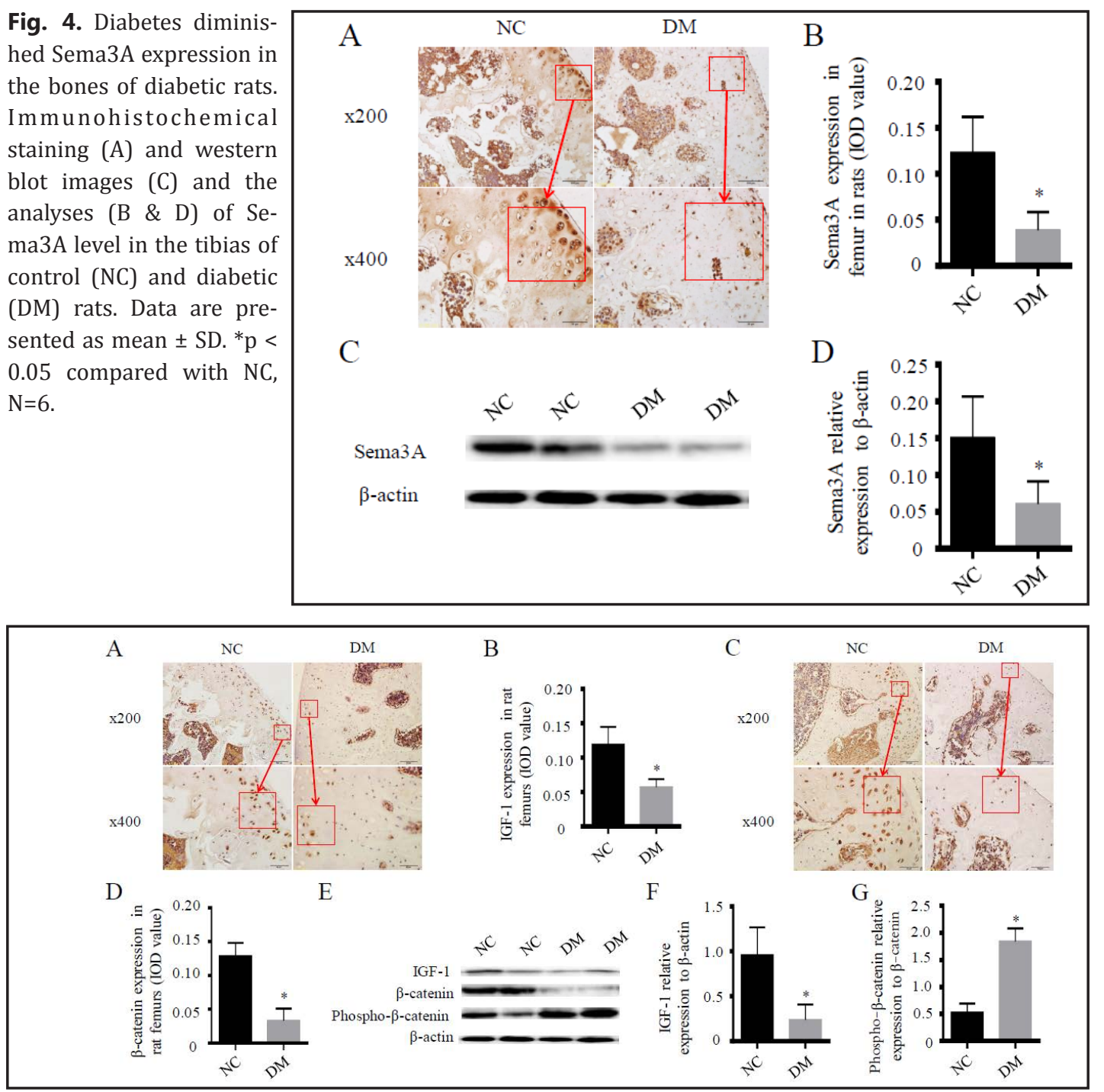

Fig. 5. Diabetes decreased the expression of IGF-1 and $\beta$-catenin as well as increased relative expression of phosphor- $\beta$-catenin to $\beta$-catenin in the bones of diabetic rats. Representative immunohistochemical and western blot images and the analyses of IGF-1(A, B, E \& F), $\beta$-catenin (C, D, E \& G) and the expression of phosphor- $\beta$-catenin to $\beta$-catenin (E \& G) in the tibias of control (NC) and diabetic (DM) rats. Data are presented as mean \pm SD. ${ }^{*} \mathrm{p}<0.05$ compared with $\mathrm{NC}, \mathrm{N}=6$.

\section{Microstructure changes in the bones of diabetic rats}

As shown in Figs. 2 and 3, micro-CT images of the femur and lumbar spine (L2) and the analyses revealed that diabetes was associated with deficits in trabecular bone microarchitecture in the femurs, such as manifested in decreased BV/TV, Tb.N, Tb.Th and Conn.D, along with increased trabecular spacing (Tb.Sp; $<<0.05$ ) and changes in trabecular shape (SMI; $p<0.05$ ). These results suggested that DM insults produced a significant reduction of bone structural strength and stiffness in the bones of diabetic rats.

The expression of Sema3A in the bones of diabetic rats

Since Sema3A contributes to bone remodeling and is deficient in osteoporosis, we subsequently examined the expression of Sema3A by IHC staining and western blot. As illustrated in Figs. 4A and B, the IHC staining intensity of Sema3A in the bones of diabetic rats was dramatically decreased compared to that of the control group $(\mathrm{p}<0.05)$. Western 


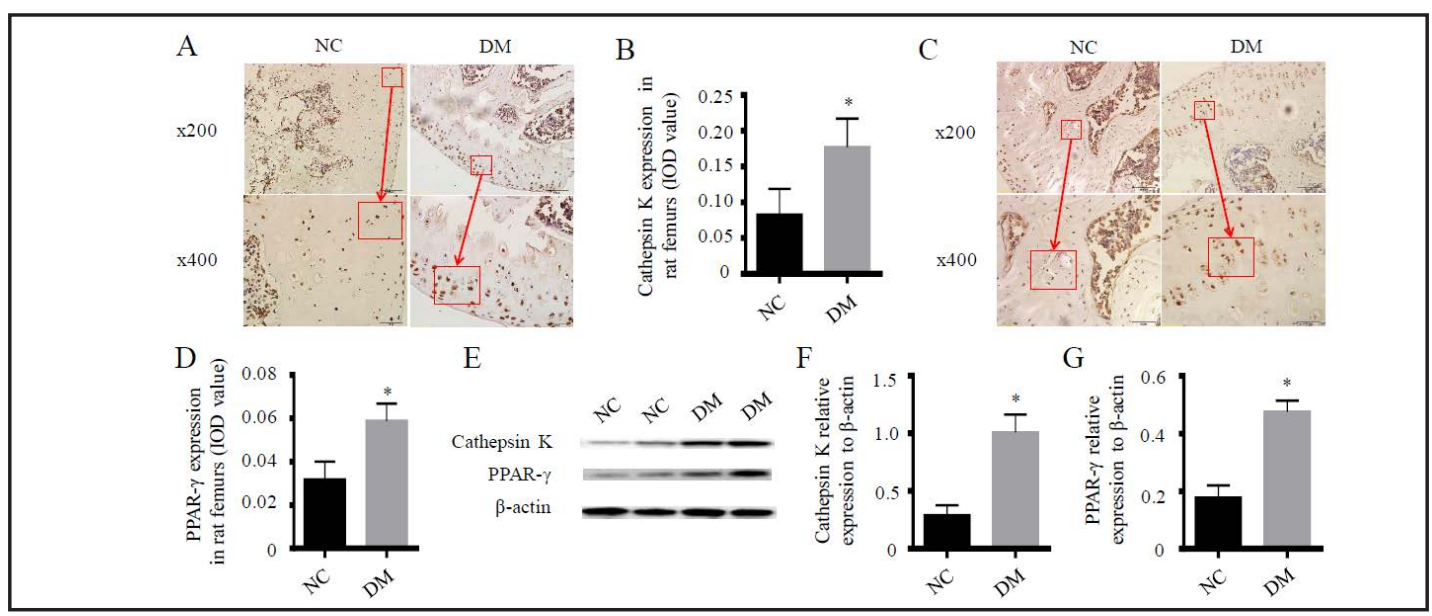

Fig. 6. Diabetes increased the expression of cathepsin $\mathrm{K}$ and PPAR $\gamma$ in rat femurs. Representative immunohistochemical and western blot images and the analyses of cathepsin K (A, B, E \& F) and PPAR $(C, D$, E \& G) levels in the tibias of control (NC) and diabetic (DM) rats. Data are presented as mean \pm SD. ${ }^{*} \mathrm{p}<0.05$ compared with NC, $\mathrm{N}=6$.

blot analysis (Figs. 4C and D) also demonstrated that Sema3A expression in the diabetic group was much lower than that in the control group $(\mathrm{p}<0.05)$. These results indicated that Sema3A was involved in the acceleration of bone loss in the development of diabetes.

The expression of IGF- 1 and $\beta$-catenin in the bones of diabetic rats

As it is known that Sema3A activates $\beta$-catenin signaling pathway [24] and IGF-1 is associated with the development of osteoporosis and $\beta$-catenin activation [5], we then determined the expression of $\beta$-catenin and IGF-1 in the bones of diabetic rats by IHC staining and western blot. As shown in Fig. 5, the expression of $\beta$-catenin and IGF- 1 in the bones of diabetic rats was markedly decreased in comparison with that in the tibias of control rats $(\mathrm{p}<0.05)$. Further, the relative expression of phosphor- $\beta$-catenin to $\beta$-catenin was significantly increased in the tibias of diabetic rats. The results suggested that lack of Sema3A could further inhibit the expression of $\beta$-catenin and IGF-1 in the bones of diabetic rats.

The expression of PPAR $\gamma$ and cathepsin $K$ in the bones of diabetic rats

Because PPAR $\gamma$ promotes mesenchymal stem cells differentiation into adipocytes rather than osteoblasts [25], and increased expression of cathepsin $\mathrm{K}$ contributes to osteoclast activation [19], we examined the expression of PPAR $\gamma$ and cathepsin $K$ in diabetic rats. IHC staining results (Figs. 6A to 6D) showed that the expression of PPAR $\gamma$ and cathepsin $\mathrm{K}$ was significantly enhanced in the cortical bones of proximal femurs in diabetic rats, when compared to the rats in the control group. These results were also verified by western blot (Figs. 6E to 6G).

\section{Discussion}

In the current study, we found the following alterations in the bones of diabetic rats. First, there is a decrease in trabecular number and volume, as well as bone formation and mineralization, and an increase in trabecular separation in the femur and lumbar spine (L2). Accordingly, the bone fragility is significantly increased and bone stiffness is decreased. Second, Sema3A expression is significantly reduced. Last but not the least, the expression of $\beta$-catenin and IGF-1 is decreased, whereas the expression of PPAR $\gamma$ and cathepsin $\mathrm{K}$ is increased. 


\section{Cellular Physiology Cell Physiol Biochem 2017;41:55-66

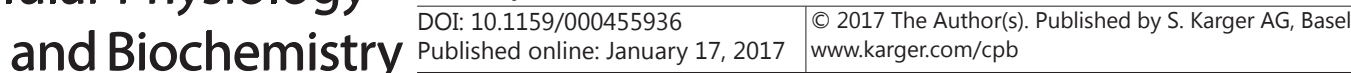

Ma et al.: Sema3A and Diabetic Osteoporosis Rats

An increasing number of studies have investigated the involvement of Sema3A in the development of diabetes and osteoporosis. Sema3A is implicated in the development of osteoporosis through regulation of bone metabolism by removal of mineralized bone and inhibition of the formation of new bone [13]. To further explore the role of Sema3A in bone remodeling, Li et al. used Sema3A embedded in matrigel to implant into the metaphysis of proximal tibias in OVX rats [17]. They found that Sema3A beneficially contributed to bone volume, bone-to-implant contact ratio and the maximal push-out force by improving periimplant bone implant, osseointegration and fixation. Similarly, Ma et al. demonstrated that naringin, the main active constituent of citrus fruits, improved bone development through increasing Sema3A expression and regulation of Wnt/ $\beta$-catenin signaling [26, 27]. Clinically, Sema3A and its receptor plexin A2 is positively associated with osteocalcin [28] and BMD [29] in postmenopausal women. However, it should be stated that Sema3A may promote the development of diabetic nephropathy [30]. In addition, Fang et al. showed that Sema3A contributed to osteogenic differentiation and improved osseointegration in HFD and STZ induced diabetic rats [18]. Our results are consistent with these previous reports that Sem $3 \mathrm{~A}$ expression is deceased in the bones of diabetic rats, which is associated with disorganized bone microstructures and increased fracture risk.

It has been demonstrated that Sema3A mediates bone protective effects through regulation of the $\beta$-catenin signaling pathway [12]. Sema3A binds to Nrp1 and subsequent activates the Wnt/ $\beta$-catenin signaling pathway which further induces osteoblast differentiation in bone formation [31]. In addition, Sema3A induces cell migration, proliferation, and odontoblastic differentiation of dental pulp stem cells, and promotes reparative dentin formation through increasing $\beta$-catenin nuclear accumulation via canonical $W n t / \beta$-catenin signaling [32]. Meanwhile, inhibition of $\beta$-catenin expression suppresses Sema3A signaling [24]. Persistent oxidative stress in diabetes impairs bone formation through inhibition of $\beta$-catenin signaling [33]. In the current study, we found that $\beta$-catenin expression in tibias and femurs in diabetic rats was significantly reduced as shown by IHC staining and western blot compared to the control animals. In conjunction with our finding of a reduction of Sema3A expression in diabetic bones and the investigations from other laboratories, the results indicate that diabetes may accelerate bone loss through inhibiting the Sema3A/ $\beta$-catenin signaling pathway. Down-regulation of Sema3A and subsequent inactivation of Wnt $/ \beta$-catenin signaling may be one of the underlying mechanisms involved in the development of diabetic rats.

In the current study, we demonstrate that the expression of $\beta$-catenin and IGF- 1 is decreased in the bones of diabetic rats which contributes to the loss of trabecular bone resulting in decreased bone stiffness and increased bone fragility. IGF-1, highly expressed in osteoblast and chondrocytes [34], exhibits its bone anabolic effect through promoting osteoblasts differentiation and bone mineralization [35]. Deficiency of IGF-1 in the course of diabetes may contribute to skeletal abnormalities and lead to impaired bone formation, and increased bone fragility [36]. Clinically, serum level of IGF-1 is positively associated with increased bone mass [37] and inversely associated with the number of vertebral fractures in postmenopausal women with T2DM [5, 38]. In addition, IGF-1 could enhance the stability of $\beta$-catenin in the bone and conditional disruption of IGF- 1 in osteocytes blocks $\beta$-catenin expression triggered by mechanical loading. IGF-1 stimulates $\beta$-catenin signaling through inhibition of glycogen synthase kinase-3 $\beta$ [39], while inhibition of $\beta$-catenin suppresses IGF1 stimulates cell proliferation and survival [40]. These experimental results suggest that Sema3A prevents bone loss via increasing expression of IGF- 1 and $\beta$-catenin, and diabetes affects bone mass and quality through disturbing the Sema3A/IGF- $1 / \beta$-catenin pathway.

PPAR $\gamma$ controls bone turnover and its activation not only suppresses bone formation but also increases bone resorption [41]. In type 1 diabetes mice, PPAR $\gamma$ increases bone marrow adiposity and decreases osteocalcin expression [25]. Increased expression of PPAR $\gamma$ negatively affects osteoblast differentiation in aging mice with diabetes which may be associated with low bone turnover and acceleration of bone loss [42]. The PPAR $\gamma$ agonists, rosiglitazone and GW1929, could increase osteoclastogenesis through enhancement of cathepsin K and 


\section{Cellular Physiology Cell Physiol Biochem 2017;41:55-66

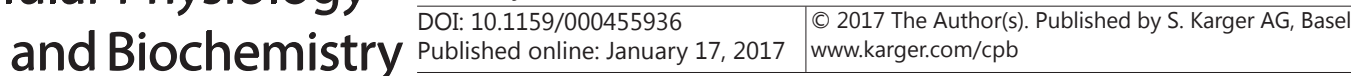 \\ Ma et al.: Sema3A and Diabetic Osteoporosis Rats}

Fig. 7. Diagram showed that diabetes could induce osteoporosis through decreasing expressions of Sema3A, IGF-1 and $\beta$-catenin as well as increasing expressions of cathepsin K and PPAR $\gamma$ in streptozotocin and high fat diet exposed rats. Further studies are still required to elucidate the relationship between Sema3A and cathepsin $\mathrm{K}$ in the course of diabetic osteoporosis.

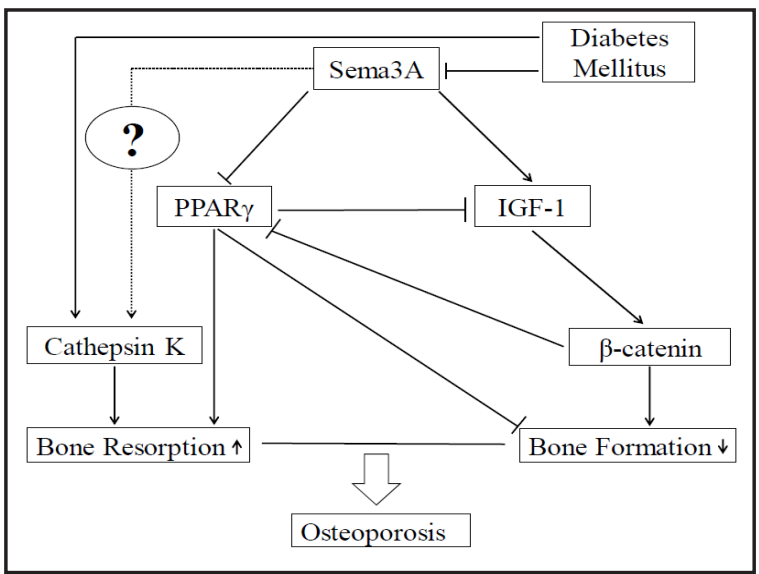

tartrate-resistant acid phosphatase expressions in bone marrow-derived macrophages [43]. Conversely, inhibition of cathepsin K also down-regulates PPAR $\gamma$ expression in HFD-induced mice [44]. In addition, $\beta$-catenin activation enhances osteoblastogenesis and suppresses adipogenesis by inhibiting PPAR $\gamma$ expression [45]. Further, PPAR $\gamma$ also down-regulates IGF1 expression, which further prevents osteoblast function and promotes osteoporosis [46]. Here, we demonstrate that the expression of PPAR $\gamma$ and cathepsin $\mathrm{K}$ is increased in diabetic rats which are associated with the acceleration of bone loss.

However, some limitations should be noted in the current study. Firstly, rats exposed to STZ and HFD results in a reduction of Sema3A and cathepsin K overexpression. Cathepsin K is not only involved in enhancing bone resorption, but also implicated in adipogenesis and glucose metabolism [47]. Inhibition of cathepsin K reduces body weight gain and glycaemia in HFD insulted rats $[44,47]$. In the current study, we found that diabetes may potentiate cathepsin K expression. Moreover, cathepsin K deficiency in mice is associated with a marked decrease in differentiated astrocytes and learning and memory deficits [48]. Sema3A is also positively involved in the regulation of the central and peripheral nervous systems. However, whether Sema3A has a direct influence on the expression of cathepsin K and the subsequent inhibition of osteoclast function still need to be further studied. Secondly, BMD values were very scattered in the bone of diabetic rats (data not shown). In type 1 diabetes, BMD values were reported to decrease[49]. However, in type 2 diabetes, BMD values were very unconclusive [50]. The reason for the discrepancy may be owed to differences in inclusion criteria, variations of diabetic complications and limited sample sizes. Moreover, bone fragility and microarchitecture may contribute to fracture risk independent of BMD in the course of diabetes.

In summary, our results demonstrate that Sema3A expression is decreased in bones of diabetic rats, which was accompanied by decreased expression of IGF- 1 and $\beta$-catenin expression as well as increased expression of cathepsin K and PPAR $\gamma$ (Fig. 7). We demonstrated for the first time that diabetes may perturb bone loss through Sema3A/IGF$1 / \beta$-catenin signaling pathway. Further studies should be undertaken to provide additional information concerning the mechanism of Sema3A involvement in the development of diabetic osteoporosis.

\section{Acknowledgements}

This work was supported by grants from National Natural Science Foundation of China (81274041, 81273995), international cooperation projects of MOE (2011DFA30920) as well as the 111 project of MOE (B07007).

\section{Disclosure Statement}

All authors declare that they have no any actual or potential conflict of interest including any financial, personal or other relationships with other people or organizations within three 


\section{Cellular Physiology Cell Physiol Biochem 2017;41:55-66 \begin{tabular}{ll|l} 
DOI: 10.1159/000455936 & O 2017 The Author(s). Published by S. Karger AG, Basel \\
www.karger.com/cpb
\end{tabular} \\ Ma et al.: Sema3A and Diabetic Osteoporosis Rats}

years of beginning the submitted work that could inappropriately influence, or be perceived to influence, their work.

\section{References}

1 Piscitelli P, Neglia C, Vigilanza A, Colao A: Diabetes and bone: biological and environmental factors. Curr Opin Endocrinol Diabetes Obes 2015;22:439-445.

2 Leidig-Bruckner G, Grobholz S, Bruckner T, Scheidt-Nave C, Nawroth P, Schneider JG: Prevalence and determinants of osteoporosis in patients with type 1 and type 2 diabetes mellitus. BMC Endocr Disord 2014;14:33.

3 Wongdee K, Charoenphandhu N: Osteoporosis in diabetes mellitus: Possible cellular and molecular mechanisms. World J Diabetes 2011;2:41-48.

4 Zhao S, Mugabo Y, Ballentine G, Attane C, Iglesias J, Poursharifi P, Zhang D, Nguyen TA, Erb H, Prentki R, Peyot ML, Joly E, Tobin S, Fulton S, Brown JM, Madiraju SR, Prentki M: alpha/beta-Hydrolase Domain 6 Deletion Induces Adipose Browning and Prevents Obesity and Type 2 Diabetes. Cell Rep 2016;14:28722888.

5 Ardawi MS, Akhbar DH, Alshaikh A, Ahmed MM, Qari MH, Rouzi AA, Ali AY, Abdulrafee AA, Saeda MY: Increased serum sclerostin and decreased serum IGF-1 are associated with vertebral fractures among postmenopausal women with type-2 diabetes. Bone 2013;56:355-362.

6 Hie M, Tsukamoto I: Increased expression of the receptor for activation of NF-kappaB and decreased runtrelated transcription factor 2 expression in bone of rats with streptozotocin-induced diabetes. Int J Mol Med 2010;26:611-618.

7 Alselami NM, Noureldeen AF, Al-Ghamdi MA, Khan JA, Moselhy SS: Bone turnover biomarkers in obese postmenopausal Saudi women with type-II diabetes mellitus. Afr Health Sci 2015;15:90-96.

8 Steneberg P, Sykaras AG, Backlund F, Straseviciene J, Soderstrom I, Edlund H: Hyperinsulinemia Enhances Hepatic Expression of the Fatty Acid Transporter Cd36 and Provokes Hepatosteatosis and Hepatic Insulin Resistance. J Biol Chem 2015;290:19034-19043.

9 Memon RA, Tecott LH, Nonogaki K, Beigneux A, Moser AH, Grunfeld C, Feingold KR: Up-regulation of peroxisome proliferator-activated receptors (PPAR-alpha) and PPAR-gamma messenger ribonucleic acid expression in the liver in murine obesity: troglitazone induces expression of PPAR-gamma-responsive adipose tissue-specific genes in the liver of obese diabetic mice. Endocrinology 2000;141:4021-4031.

10 Rosen ED, Sarraf P, Troy AE, Bradwin G, Moore K, Milstone DS, Spiegelman BM, Mortensen RM: PPAR gamma is required for the differentiation of adipose tissue in vivo and in vitro. Mol Cell 1999;4:611-617.

11 Liang Y, Wang W, Huang J, Tan H, Liu T, Shang C, Liu D, Guo L, Yao S: Potential Role of Semaphorin 3A and Its Receptors in Regulating Aberrant Sympathetic Innervation in Peritoneal and Deep Infiltrating Endometriosis. PLoS One 2015;10:e0146027.

12 Hayashi M, Nakashima T, Taniguchi M, Kodama T, Kumanogoh A, Takayanagi H: Osteoprotection by semaphorin 3A. Nature 2012;485:69-74.

13 Xu R: Semaphorin 3A: A new player in bone remodeling. Cell Adh Migr 2014;8:5-10.

14 Yamashita T, Takahashi N, Udagawa N: New roles of osteoblasts involved in osteoclast differentiation. World J Orthop 2012;3:175-181.

15 Hu H, Chen M, Dai G, Du G, Wang X, He J, Zhao Y, Han D, Cao Y, Zheng Y, Ding D: An Inhibitory Role of Osthole in Rat MSCs Osteogenic Differentiation and Proliferation via Wnt/beta-Catenin and Erk1/2-MAPK Pathways. Cell Physiol Biochem 2016;38:2375-2388.

16 Sarvari M, Kallo I, Hrabovszky E, Solymosi N, Liposits Z: Ovariectomy and subsequent treatment with estrogen receptor agonists tune the innate immune system of the hippocampus in middle-aged female rats. PLoS One 2014;9:e88540.

17 Li Y, He D, Liu B, Hu J: SEMA3A suspended in matrigel improves titanium implant fixation in ovariectomized rats. J Biomed Mater Res B Appl Biomater DOI:10.1002/jbm.b.33730.

18 Fang K, Song W, Wang L, Xu X, Tan N, Zhang S, Wei H, Song Y: Semaphorin 3A-modified adipose-derived stem cell sheet may improve osseointegration in a type 2 diabetes mellitus rat model. Mol Med Rep 2016;14:2449-2456. 


\section{Cellular Physiology Cell Physiol Biochem 2017;41:55-66 \begin{tabular}{l|l|l}
\hline DOI: 10.1159/000455936 & 2017 The Author(s). Published by S. Karger AG, Basel
\end{tabular} and Biochemistry Published online: January 17, 2017 www.karger.com/cpb \\ Ma et al.: Sema3A and Diabetic Osteoporosis Rats}

19 Guo Y, Wang L, Ma R, Mu Q, Yu N, Zhang Y, Tang Y, Li Y, Jiang G, Zhao D, Mo F, Gao S, Yang M, Kan F, Ma Q Fu M, Zhang D: JiangTang XiaoKe granule attenuates cathepsin K expression and improves IGF-1 expression in the bone of high fat diet induced KK-Ay diabetic mice. Life Sci 2016;148:24-30.

20 Mathews S, Mathew SA, Gupta PK, Bhonde R, Totey S: Glycosaminoglycans enhance osteoblast differentiation of bone marrow derived human mesenchymal stem cells. J Tissue Eng Regen Med 2014;8:143-152.

21 Coulson-Thomas YM, Coulson-Thomas VJ, Norton AL, Gesteira TF, Cavalheiro RP, Meneghetti MC, Martins JR, Dixon RA, Nader HB: The identification of proteoglycans and glycosaminoglycans in archaeological human bones and teeth. PLoS One 2015;10:e0131105.

22 Du G, Song Y, Wei L, Li L, Wang X, Xu Q, Zhan H, Cao Y, Zheng Y, Ding D: Osthole Inhibits Proliferation and Induces Catabolism in Rat Chondrocytes and Cartilage Tissue. Cell Physiol Biochem 2015;36:2480-2493.

23 Song L, Zhao J, Zhang X, Li H, Zhou Y: Icariin induces osteoblast proliferation, differentiation and mineralization through estrogen receptor-mediated ERK and JNK signal activation. Eur J Pharmacol 2013;714:15-22.

24 Hida T, Yamashita N, Usui H, Nakamura F, Sasaki Y, Kikuchi A, Goshima Y: GSK3beta/axin-1/beta-catenin complex is involved in semaphorin3A signaling. J Neurosci 2012;32:11905-11918.

25 Botolin S, McCabe LR: Inhibition of PPARgamma prevents type I diabetic bone marrow adiposity but not bone loss. J Cell Physiol 2006;209:967-976.

26 Ma X, Lv J, Sun X, Ma J, Xing G, Wang Y, Sun L, Wang J, Li F, Li Y, Zhao Z: Naringin ameliorates bone loss induced by sciatic neurectomy and increases Semaphorin 3A expression in denervated bone. Sci Rep 2016;6:24562.

27 Wang D, Ma W, Wang F, Dong J, Wang D, Sun B, Wang B: Stimulation of Wnt/beta-Catenin Signaling to Improve Bone Development by Naringin via Interacting with AMPK and Akt. Cell Physiol Biochem 2015;36:1563-1576.

28 Liu DM, Lu N, Zhao L, Zhang MJ, Tao B, Xuan Y, Sun LH, Zhao HY, Wang WQ Liu JM, Ning G: Serum Sema3A is in a weak positive association with bone formation marker osteocalcin but not related to bone mineral densities in postmenopausal women. J Clin Endocrinol Metab 2014;99:E2504-2509.

29 Hwang JY, Lee JY, Park MH, Kim KS, Kim KK, Ryu HJ, Lee JK, Han BG, Kim JW, Oh B, Kimm K, Park BL, Shin HD, Kim TH, Hong JM, Park EK, Kim DJ, Koh JM, Kim GS, Kim SY: Association of PLXNA2 polymorphisms with vertebral fracture risk and bone mineral density in postmenopausal Korean population. Osteoporos Int 2006;17:1592-1601.

30 Aggarwal PK, Veron D, Thomas DB, Siegel D, Moeckel G, Kashgarian M, Tufro A: Semaphorin3a promotes advanced diabetic nephropathy. Diabetes 2015;64:1743-1759.

31 Krishnan V, Bryant HU, Macdougald OA: Regulation of bone mass by Wnt signaling. J Clin Invest 2006;116:1202-1209.

32 Yoshida S, Wada N, Hasegawa D, Miyaji H, Mitarai H, Tomokiyo A, Hamano S, Maeda H: Semaphorin 3A Induces Odontoblastic Phenotype in Dental Pulp Stem Cells. J Dent Res DOI:10.1177/0022034516653085.

33 Mori K, Kitazawa R, Kondo T, Mori M, Hamada Y, Nishida M, Minami Y, Haraguchi R, Takahashi Y, Kitazawa $\mathrm{S}$ : Diabetic osteopenia by decreased beta-catenin signaling is partly induced by epigenetic derepression of sFRP-4 gene. PLoS One 2014;9:e102797.

34 Kawai M, Rosen CJ: The insulin-like growth factor system in bone: basic and clinical implications. Endocrinol Metab Clin North Am 2012;41:323-333, vi.

35 Sheng MH, Lau KH, Baylink DJ: Role of Osteocyte-derived Insulin-Like Growth Factor I in Developmental Growth, Modeling, Remodeling, and Regeneration of the Bone. J Bone Metab 2014;21:41-54.

36 Sroga GE, Wu PC, Vashishth D: Insulin-like growth factor 1, glycation and bone fragility: implications for fracture resistance of bone. PLoS One 2015;10:e0117046.

37 Fowlkes JL, Nyman JS, Bunn RC, Jo C, Wahl EC, Liu L, Cockrell GE, Morris LM, Lumpkin CK, Jr., Thrailkill KM: Osteo-promoting effects of insulin-like growth factor I (IGF-I) in a mouse model of type 1 diabetes. Bone 2013;57:36-40.

38 Ashpole NM, Herron JC, Mitschelen MC, Farley JA, Logan S, Yan H, Ungvari Z, Hodges EL, Csiszar A, Ikeno Y, Humphrey MB, Sonntag WE: IGF-1 Regulates Vertebral Bone Aging Through Sex-Specific and TimeDependent Mechanisms. J Bone Miner Res 2016;31:443-454. 


\section{Cellular Physiology Cell Physiol Biochem 2017;41:55-66 \begin{tabular}{l|l|l} 
DOI: 10.1159/000455936 & $\begin{array}{l}\text { O 2017 The Author(s). Published by S. Karger AG, Basel } \\
\text { www.karger.com/cpb }\end{array}$
\end{tabular} \\ Ma et al.: Sema3A and Diabetic Osteoporosis Rats}

39 Desbois-Mouthon C, Cadoret A, Blivet-Van Eggelpoel MJ, Bertrand F, Cherqui G, Perret C, Capeau J: Insulin and IGF-1 stimulate the beta-catenin pathway through two signalling cascades involving GSK-3beta inhibition and Ras activation. Oncogene 2001;20:252-259.

40 Ye P, Hu Q Liu H, Yan Y, D'Ercole A J: beta-catenin mediates insulin-like growth factor-I actions to promote cyclin D1 mRNA expression, cell proliferation and survival in oligodendroglial cultures. Glia 2010;58:10311041.

41 Wan Y: PPARgamma in bone homeostasis. Trends Endocrinol Metab 2010;21:722-728.

42 Portal-Nunez S, Ardura JA, Lozano D, Bolivar OH, Lopez-Herradon A, Gutierrez-Rojas I, Proctor A, van der Eerden B, Schreuders-Koedam M, van Leeuwen J, Alcaraz MJ, Mulero F, de la Fuente M, Esbrit P: Adverse Effects of Diabetes Mellitus on the Skeleton of Aging Mice. J Gerontol A Biol Sci Med Sci 2016;71:290-299.

43 Nakanishi A, Tsukamoto I: n-3 polyunsaturated fatty acids stimulate osteoclastogenesis through PPARgamma-mediated enhancement of c-Fos expression, and suppress osteoclastogenesis through PPARgamma-dependent inhibition of NFkB activation. J Nutr Biochem 2015;26:1317-1327.

44 Han J, Wei L, Xu W, Lu J, Wang C, Bao Y, Jia W: CTSK inhibitor exert its anti-obesity effects through regulating adipocyte differentiation in high-fat diet induced obese mice. Endocr J 2015;62:309-317.

45 Xu C, Wang J, Zhu T, Shen Y, Tang X, Fang L, Xu Y: Cross-Talking Between PPAR and WNT Signaling and its Regulation in Mesenchymal Stem Cell Differentiation. Curr Stem Cell Res Ther 2016;11:247-254.

46 Lecka-Czernik B, Ackert-Bicknell C, Adamo ML, Marmolejos V, Churchill GA, Shockley KR, Reid IR, Grey A, Rosen CJ: Activation of peroxisome proliferator-activated receptor gamma (PPARgamma) by rosiglitazone suppresses components of the insulin-like growth factor regulatory system in vitro and in vivo. Endocrinology 2007;148:903-911.

47 Yang M, Sun J, Zhang T, Liu J, Zhang J, Shi MA, Darakhshan F, Guerre-Millo M, Clement K, Gelb BD, Dolgnov G, Shi GP: Deficiency and inhibition of cathepsin K reduce body weight gain and increase glucose metabolism in mice. Arterioscler Thromb Vasc Biol 2008;28:2202-2208.

48 Dauth S, Schmidt MM, Rehders M, Dietz F, Kelm S, Dringen R, Brix K: Characterisation and metabolism of astroglia-rich primary cultures from cathepsin K-deficient mice. Biol Chem 2012;393:959-970.

49 Jackuliak P, Payer J: Osteoporosis, Fractures, and Diabetes. Int J Endocrinol 2014;2014:10.

50 Oei L, Zillikens MC, Dehghan A, Buitendijk GH, Castano-Betancourt MC, Estrada K, Stolk L, Oei EH, van Meurs JB, Janssen JA, Hofman A, van Leeuwen JP, Witteman JC, Pols HA, Uitterlinden AG, Klaver CC, Franco $\mathrm{OH}$, Rivadeneira F: High bone mineral density and fracture risk in type 2 diabetes as skeletal complications of inadequate glucose control: the Rotterdam Study. Diabetes Care 2013;36:1619-1628. 